\title{
IMPACTO DEL DESARROLLO DE HABILIDADES DE PENSAMIENTO CRÍTICO EN LA COMPRENSIÓN LECTORA DE ESTUDIANTES DE ENSEÑANZA BÁSICA
}

\section{IMPACT OF THE DEVELOPMENT OF CRITICAL THINKING SKILLS ON THE READING COMPREHENSION OF ELEMENTARY SCHOOL STUDENTS}

\author{
Ivette Doll Castillo \\ Universidad de Playa Ancha, Chile. \\ Ivette.doll@upla.cl \\ Claudio Parra Vásquez \\ Universidad Andrés Bello, Chile. \\ cparravasquez@gmail.com
}

\begin{abstract}
Resumen:
La investigación pretendió determinar, a través de un estudio cuasiexperimental con grupo control, los efectos globales y diferenciales de una experiencia programática de desarrollo de habilidades del pensamiento crítico en los niveles de desempeño de comprensión lectora de 57 estudiantes de octavo año básico. El programa de intervención se diseñó en función de los postulados teóricos y metodológicos de autores como Mathew Lipman, Richard Paul y Linda Elder, quienes le otorgan al cuestionamiento de tipo socrático un rol fundamental en la evaluación y análisis del razonamiento que tiene lugar en el proceso de comprensión lectora. El estudio revela que solo el grupo experimental evidencia un cambio positivo y estadísticamente significativo en el nivel global de desempeño de comprensión lectora y en seis de las siete áreas evaluadas.
\end{abstract}

Palabras Clave: Habilidades de pensamiento crítico, comprensión lectora, proceso lector, interrogación textual y comprensión crítica.

\begin{abstract}
:
The research aimed to determine, through a quasi-experimental study, the overall and differential effects of a programmatic experience in the development of critical thinking skills on reading comprehension levels of performance of 57 eight-graded elementary school students. The intervention program was designed based on the theoretical and practical postulates of authors such as Mathew Lipman, Richard Paul and Linda Elder, who give to the Socratic questioning a fundamental role in the evaluation and analysis of the reasoning that takes place in the reading comprehension process. The study unveils that only the experimental group shows a positive and statistically significant change in the overall level of reading comprehension performance and in six of the seven areas evaluated.
\end{abstract}

Key Words: Critical thinking skills, reading comprehension, reading process, textual questioning, critical comprehension. 
Artículo. Ivette Doll, Claudio Parra. "Impacto del desarrollo de habilidades de pensamiento crítico en la comprensión lectora de estudiantes de Enseñanza Básica"

Recibido: 29 de septiembre de 2021

Aceptado: 18 de noviembre de 2021

\section{Introducción}

Los sistemas a través de los cuales se evalúa la calidad de la educación que se imparte en Chile revelan, en promedio, un bajo nivel de desarrollo de la competencia lectora de los escolares que cursan la enseñanza básica y media. ¿Por qué los escolares chilenos exhiben estos preocupantes resultados? y ¿por qué fracasan los intentos del estado por mejorar significativamente los niveles de desarrollo de esta competencia? Más allá de las explicaciones causales, originadas en las marcadas diferencias socioeconómicas que afectan la calidad de la educación chilena, Cairney (35) nos ofrece una posible hipótesis; válida, por cierto, para cualquier realidad educativa. El autor señala que las escuelas basan la enseñanza de estrategias de comprensión lectora en laboratorios y fichas de lectura, a las cuales les siguen una serie de preguntas para comprobar la comprensión. Este enfoque resulta poco útil para los estudiantes y escasamente apunta a la creación personal de significados; solo se centra en la reproducción de ideas que otros ya pensaron. La creación personal de significados exige a los estudiantes el logro de un nivel crítico de lectura (reflexionar y evaluar en PISA y reflexionar en el SIMCE), porque solo en ese nivel se pueden liberar de las ataduras que les impone la lectura pasiva y reproductiva de un texto. Alcanzarán también el nivel de emancipación cuando usen la comprensión como un medio que estimule el pensamiento y cuando el pensamiento afecte, a la inversa, sus niveles de comprensión textual.

El tipo de pensamiento que mejor se relaciona con la comprensión lectora es el pensamiento crítico. Y lo es por su condición de director de todos los otros tipos de pensamiento que afectan la capacidad adaptativa del ser humano. En él hallamos una poderosa herramienta para mejorar todos los procesos que involucran precisamente el sublime acto del pensamiento. La comprensión lectora es un acto de pensamiento. Así lo entendió Mathew Lipman, quien creó el Programa Filosofía para Niños hacia fines de la década del 60. En el marco de este programa, Lipman ideó un conjunto de novelas para desarrollar la autonomía de pensamiento de niños y niñas (también jóvenes y adultos), bajo 
la convicción de que la comprensión lectora y el pensamiento se afectan recíprocamente (Lipman, La Filosofía 70). En esa misma línea trabajó Richard Paul y lo hace actualmente Lindar Elder, su más estrecha colaboradora. Estos autores desarrollaron un modelo para potenciar el pensamiento crítico en función de estándares, virtudes intelectuales, y elementos constitutivos de la dimensión analítica y evaluativa del pensamiento. La prolífica producción de obras de Paul y Elder ilustran ampliamente la manera cómo aplicar el modelo a la comprensión de textos.

Diversas investigaciones han demostrado la correlación que existe entre ambas variables o el positivo impacto que tiene el desarrollo programático de habilidades de pensamiento crítico en la comprensión lectora (Diella y Adriansyaah 136-139, Ardhian et al. 112-116, por citar solo algunos). Lamentablemente, el grueso de estos estudios se sitúa en los últimos años de la enseñanza secundaria o en los primeros años de la educación superior (Gutiérrez 309-318, Mohseni et al. 101-127, entre otros). En virtud de la brecha indagativa que se genera en poblaciones de estudiantes de cursos inferiores, en esta investigación se pretendió dar respuesta a las siguientes interrogantes: ¿qué efectos tiene el desarrollo programático de las habilidades de pensamiento crítico en el nivel de desempeño global de comprensión lectora de estudiantes que cursan enseñanza básica? y ¿qué efectos diferenciales tiene el desarrollo de habilidades de pensamiento crítico en el nivel de desarrollo de las distintas habilidades de comprensión lectora? Se estimó que las respuestas a estas interrogantes, en el contexto de una investigación cuasiexperimental, podían iluminar el debate en torno a la manera cómo mejorar la comprensión lectora de los estudiantes chilenos.

\section{Marco teórico}

\subsection{La comprensión lectora}

El concepto de comprensión lectora está ligado a los conceptos de lectura, comprensión de textos y competencia lectora. Aun cuando estos cuatro conceptos se usan indistintamente para referirse al acto de interpretar los mensajes que un escritor elabora para un lector, cada uno de ellos comprende rasgos que le son propios. Según Núñez (117), la 
Artículo. Ivette Doll, Claudio Parra. "Impacto del desarrollo de habilidades de pensamiento crítico en la comprensión lectora de estudiantes de Enseñanza Básica"

161

palabra lectura tiene dos acepciones: la primera se refiere al acto de decodificar (en el contexto del aprender a leer) y, la segunda, a la interpretación del lenguaje escrito. Parodi et al. concuerdan con la idea de que saber leer no es equivalente solo a un proceso de decodificación, sino que "implica un esfuerzo por la construcción de significado; todo ello, sobre la base de diversos elementos funcionales y contextuales que son relacionados por medio de la cognición del lector con el texto que está leyendo (Saber 65). Para los autores, cuatro son los factores que determinan este macroproceso de construcción interpretativa que realiza un lector: la información del texto escrito, los conocimientos previos, el objetivo de lectura y las posibles demandas del medio social que mediatizan la necesidad de comprensión.

En el marco de la prueba PISA, a la comprensión lectora se le atribuye el carácter de competencia (reading literacy) y se la define como "la comprensión, el uso, la evaluación, la reflexión y el compromiso con los textos, a fin de alcanzar las propias metas, desarrollar el conocimiento y el potencial personal y participar en la sociedad. (OCDE 23).

La actividad de comprensión lectora ha estado ligada principalmente a la necesidad que tienen las personas de asignarle sentido a los textos escritos. De hecho, aprender a leer y escribir sigue siendo uno de los principales propósitos educativos en las escuelas. Pero el concepto texto no solo se relaciona con un conjunto coherente de ideas escritas o de naturaleza verbal, sino que también con un conjunto de ideas que se pueden expresar a través de distintos sistemas semióticos o repertorio de signos que se articulan a partir de variados principios de organización. Parodi (Multisemiosis 37) señala que un texto escrito es intrínsecamente multisemiótico, toda vez que en él se pueden orquestar diversos sistemas semióticos como el gráfico (fotografías, gráficos, diagramas, tablas y bocetos), el verbal (palabras, frases, oraciones), el tipográfico (forma y color de las letras) y el matemático (números, letras, operadores y signos de puntuación). En consecuencia, comprender un texto exige desentrañar, primero, el sentido de cada uno de los signos o de los significados al interior de cada sistema semiótico y, luego, determinar los sentidos que derivan de las relaciones entre cada uno de los sistemas que configuran la realidad textual. 
Si bien es cierto en esta investigación se reconocen las particularidades de cada concepto y la manera cómo se complementan, se prefiere el término comprensión lectora por tratarse de una locución universalmente reconocida en los contextos educativos.

Hay consenso en que existen tres tipos de modelos que explican la manera cómo se realiza el proceso lector. El primero de ellos, denominado de abajo-arriba, postula, en general, que lo más importante en el proceso lector es la decodificación lineal y automatizada de la información textual ; el segundo, denominado de arriba-abajo, plantea que el proceso lector está dirigido por la información que se halla almacenada en la memoria; finalmente, el modelo interactivo postula que la comprensión lectora es el producto de la interacción entre los conocimientos previos del lector y la información del texto. En el modelo interactivo de Kintsch (811) se propone que, en el proceso lector, se construyen dos tipos de estructuras textuales: la microestructura y la macroestructura. La microestructura está formada por todas las proposiciones del texto vinculadas entre sí. Las proposiciones corresponden a la representación lógica de las frases y oraciones del texto. Las proposiciones se generan desde la información textual más alguna información de la memoria de largo plazo. La microestructura se construye mediante cortos ciclos de procesamiento de palabras, frases y oraciones. Por su parte, la macroestructura combina el conocimiento previo y las inferencias que realiza el lector con la microestructura del texto, es decir, con el conjunto de proposiciones incardinadas. A partir de estas dos estructuras, los lectores forman dos tipos de representaciones diferenciadas. A la primera de ellas se le llama 'texto base' y corresponde a la representación más fiel al texto leído. A su vez, la representación de la manera cómo han entendido los lectores el texto corresponde al 'modelo situacional', compuesto por las proposiciones derivadas del texto base y por las proposiciones elaboradas en la memoria a largo plazo. El modelo situacional integra el conocimiento previo del lector con la información del texto. Conforme a los postulados de Parodi (La teoría 148), es posible concluir que el proceso lector no termina cuando se elabora el texto base y el modelo situacional. Según el autor, la comprensión de un texto llega a su fin cuando el lector acredita o comunica aquello que ha comprendido a través de algún modo semiótico.

Siguiendo la línea argumentativa del modelo de Kintsch, Sánchez et al. establecen tres niveles de comprensión: un nivel de comprensión superficial, un nivel de comprensión profunda y un nivel de comprensión crítica o reflexiva (63). El primer nivel de comprensión 
Artículo. Ivette Doll, Claudio Parra. "Impacto del desarrollo de habilidades de pensamiento crítico en la comprensión lectora de estudiantes de Enseñanza Básica"

permite al lector, por ejemplo, resumir un texto, parafrasearlo y recordarlo, pero usando solo la información contenida en el texto; en este caso, las ideas que pudiese aportar el lector no añaden información nueva al texto; en el mejor de los casos, sus ideas integran o sintetizan información literal. En el nivel de comprensión profunda, el lector puede "comprender el mundo o situación a la que se refiere el texto" (63). Para lograr esta meta, debe activar sus conocimientos previos y llevar a cabo algún proceso de integración (elaboración de inferencias e interpretaciones). El nivel de comprensión crítica, el lector puede resolver problemas de inconsistencias locales y globales y puede "juzgar la calidad del texto, sus fines y el grado en el que dichos fines se alcanzan" (63).

\subsection{El pensamiento crítico}

El pensamiento crítico es una de las competencias fundamentales para participar activamente en las sociedades democráticas que usan la información y el conocimiento como fuente de desarrollo. Gracias a esta competencia podemos evaluar y analizar la calidad de nuestro pensamiento y de quienes nos rodean. Este es un propósito de primer orden, toda vez que gran parte de nuestro pensamiento "está sesgado, distorsionado, parcializado, desinformado o completamente prejuiciado" (Paul y Elder, Critical Thinking. Concepts 2).

En su condición de competencia esencial, el pensamiento crítico se constituye en una fuerza liberadora que nos permite pensar de manera autónoma, para evitar las ataduras que, a veces, nos imponen las doctrinas, las ideologías, los medios de comunicación y todos los mentefactos creados por la humanidad como instrumento de manipulación, de opresión y de subyugación. Facione lo define como "juicio deliberado y autorregulado que se usa para interpretar, analizar, evaluar e inferir; así como para explicar las consideraciones conceptuales, metodológicas, de criterio, de evidencias y contextuales en las cuales se basa el juicio" (4). Esta definición tiene tres aspectos que deben ser destacados. En primer lugar, por 'deliberado' entiende que los razonamientos son intencionados, es decir, implican una disposición especial o una voluntad inquisitiva que orienta el acto del pensamiento. El término ‘autorregulado' se refiere a una consideración de carácter metacognitiva que permite al pensamiento crítico actuar como un director que orquesta y evalúa al resto de los 
pensamientos. Por último, la definición explicita las habilidades que se sitúan en la base de este tipo de pensamiento.

Paul y Elder señalan que el pensamiento crítico es el "monitoreo sistemático del pensamiento con el propósito de mejorarlo" (Critical Thinkin.Tools 25). La mejora, conforme a estos autores, se realiza en función de procesos de análisis y evaluación. Desde la perspectiva analítica, proponen los siguientes seis elementos constitutivos, a través de los cuales se puede analizar cualquier pensamiento: determinar el propósito del pensamiento y la pregunta central que se intenta responder, identificar la información (datos, hechos, observaciones, experiencias, evidencias) a partir de la cual se elaboran las ideas, identificar las conclusiones a las cuales se llega, las soluciones que se proponen, las interpretaciones e inferencias, identificar los principales conceptos a partir de los cuales se elaboran los principios, leyes, axiomas, teorías, definiciones y modelos, determinar los supuestos del pensamiento, aquello que se acepta como dado, identificar las implicancias del pensamiento y determinar los puntos de vista, marcos de referencia, orientaciones. Para evaluar el pensamiento y los juicios que de él se derivan, los autores definen un conjunto de seis criterios o estándares de razonamiento: claridad, precisión, exactitud, pertinencia, relevancia, profundidad amplitud y justicia. La evaluación implica activar procesos metacognitivos (autorregulación y automonitoreo, por ejemplo) con la finalidad de autocorregir los pensamientos que no adhieren a los criterios que definen su calidad.

\subsection{Relación entre comprensión de lectura y pensamiento crítico}

Tanto la comprensión de lectura como el pensamiento crítico son macroprocesos de pensamiento en los que se conjugan un conjunto de habilidades a través de las cuales se procesa y produce información y conocimiento. Las habilidades cognitivas fundamentales que comparten ambos macroprocesos de pensamiento son las siguientes: interpretación, análisis, evaluación, inferencia, explicación y autorregulación (Facione 2). Al respecto, es relevante indicar que estas habilidades fueron consideradas constitutivas del pensamiento crítico en el proyecto Delphi, un esfuerzo internacional que clarificó y estandarizó la aproximación conceptual a este tipo de pensamiento. En el marco de la comprensión lectora, la interpretación y la inferencia son claves para elaborar la macroestructura y el modelo 
Artículo. Ivette Doll, Claudio Parra. "Impacto del desarrollo de habilidades de pensamiento crítico en la comprensión lectora de estudiantes de Enseñanza Básica"

situacional a través de procesos de construcción e integración (kintsch 809-834). La evaluación, por su parte, es una habilidad clave para valorar, entre otras cosas, la credibilidad y la calidad de los enunciados, de las fuentes textuales, de los argumentos que se pueden esgrimir en favor de una tesis, de los supuestos, de las suposiciones que son parte del entramado textual y de los juicios que derivan del proceso de comprensión textual. A partir de los planteamientos de Paul y Elder (Critical Thinking, Tools 59-65), es posible señalar que el análisis ayuda a identificar los siguientes aspectos de un texto: propósito del autor, información que opera como respaldo de ideas o juicios, conclusiones, conceptos clave, supuestos, implicancias, puntos de vista y preguntas centrales que el autor desea responder directa o indirectamente. La explicación es una habilidad que el lector usa cuando debe acreditar su comprensión o los razonamientos que se derivan del proceso de comprensión textual. Es importante recordar que para Parodi el proceso de comprensión se completa solo si el lector es capaz de dar cuenta de su comprensión a través de algún modo semiótico ( $L a$ Teoría 148). La autorregulación es uno de los componentes fundamentales de lo que se denomina metacognición (Flavell 232), o metacomprensión (Peronard et al. 151). Dada la naturaleza autocorrectiva y autorregulada de la comprensión textual y del pensamiento crítico (Facione 3; Lipman, Pensamiento 287; Paul y Elder, Critical Thinking, Tools xix), la metacognición es una de las habilidades claves para entender la relación entre ambos macroprocesos de pensamiento.

Existen varios estudios que abordan la relación entre pensamiento crítico y comprensión lectora y que concluyen, por una parte, una positiva y alta correlación entre ambas variables (Fernández et al. 22-26; Arslan 3-6; Cakici 119-12 ; Magno 145-152 y Din 6-10 ) y, por otra, un positivo efecto de la enseñanza de estrategias y técnicas de pensamiento crítico en el desarrollo de las habilidades de comprensión lectora (Esmaeli y Motaghizadeh 101-127; Rouijel y Bouziane 120-122). 


\subsection{El cuestionamiento como fundamento del desarrollo del pensamiento crítico y de la comprensión de lectura}

El cuestionamiento es una de las claves del pensamiento. Para Paul y Elder "no es posible ser un buen pensador sino se es un buen cuestionador (Critical Thinking. Tools 124). Y lo es, porque gracias a esta habilidad podemos buscar respuestas a millones de interrogantes que se originan en la curiosidad, el afán de conocimiento, la incertidumbre, la duda, el deseo de autoafirmación y tantos otros propósitos inherentes a nuestra naturaleza humana. Pero el cuestionamiento tiene en las respuestas a su peor enemigo toda vez que la mediatez de la certidumbre y el adoctrinamiento del pensamiento limitan la necesidad de abrirse al mundo de manera prístina, pueril y original, tal como lo hacen los niños y las niñas en su primera infancia. Eso es precisamente lo que sucede en la escuela; nuestros estudiantes van a buscar respuestas, porque en ellas está el saber y ese saber automatizado es lo que se debe transmitir. Hoy, el saber está constituido por respuestas, no por preguntas (Freire y Faúndez 69).

Aun cuando la habilidad del cuestionamiento está en el subconsciente de niños y niñas (porque fue una habilidad inherente a su condición de infante), es necesario enseñarles a preguntar de manera autónoma. Para ello es menester partir de sus propias necesidades y de sus propias experiencias. En el contexto de la comprensión de un texto, ello implica que el lector cuestione el texto a partir de sus propósitos, intereses, conocimientos previos y cosmovisiones en torno a las temáticas que sostienen la estructura textual.

Del cúmulo de taxonomías del cuestionamiento, especial interés revisten las preguntas de naturaleza socrática. Paul y Elder plantean que estas preguntas tienen una estrecha relación con el pensamiento crítico ( Critical Thinking. The Art 36), ya que proveen al pensante una voz interior de la razón para monitorear, evaluar y reparar, en una dirección más racional, sus pensamientos, sentimientos y acciones (Paul y Elder, Critical Thinking. Tools 130). De ello se colige que este tipo de preguntas ayuda a mejorar la calidad del pensamiento del pensante. Asumiendo que la comprensión de textos se traduce en una búsqueda del significado o sentido, el cuestionamiento socrático es, precisamente, un medio muy poderoso para asignar significado o coherencia al entramado textual; por ello, 
Artículo. Ivette Doll, Claudio Parra. "Impacto del desarrollo de habilidades de pensamiento crítico en la comprensión lectora de estudiantes de Enseñanza Básica"

investigadores como Cojocariu y Butnaru han usado el método socrático del cuestionamiento para mejorar la comprensión lectora y el pensamiento crítico de los estudiantes (23 y 24).

\section{Marco metodológico}

\subsection{Metodología de la investigación}

La investigación se enmarcó en un estudio cuasiexperimental con diseño de grupo control no equivalente (Cohen et al. 283-284). La población correspondió a estudiantes de octavo año básico que cursaban el nivel en colegios subvencionados de la Provincia de Marga-Marga, Región de Valparaíso. El muestreo fue de tipo no probabilístico y por conveniencia (Cohen et al. 113-114). La muestra quedó conformada por 110 estudiantes de octavo básico de dos colegios subvencionados de la población objeto, con edades comprendidas entre los 13 y 14 años. Del total, 53 estudiantes del primer centro educativo conformaron el grupo de control; los 57 restantes, pertenecientes al segundo centro, configuraron el grupo experimental. Se contemplaron tres variables de estudio: dos dependientes y otra independiente. La primera variable dependiente se denominó 'nivel de desempeño global en comprensión lectora' y se definió como el grado de logro general alcanzado por los estudiantes objeto de estudio después de rendir las pruebas de comprensión lectora de $8^{\circ}$ grado de la batería evaluativa CL-PT (Fundación Arauco, Medina y Gajardo 3-16), compuestas por reactivos que evalúan las habilidades que se conjugan en ese macroproceso de pensamiento, a través del cual el lector construye intencionadamente una interpretación de los significados elaborados por un escritor, basándose en la información textual y en sus conocimientos previos, de acuerdo a un objetivo de lectura y a las posibles demandas del medio social (Parodi, La Teoría 154 ). La prueba CL-PT evalúa siete rasgos o dimensiones de comprensión lectora: comprensión literal, comprensión inferencial, comprensión crítica, comprensión metacognitiva, identificación de estructurales textuales y funciones, reorganización de la información y manejo del código. La batería define cinco niveles de desempeño, conforme a los porcentajes de logro exhibido por los estudiantes: muy desarrollado (81-100\%), desarrollo satisfactorio (61-80\%), en desarrollo 
(41-60\%), emergente (21-40\%) y no desarrollado (0-20\%). La segunda variable dependiente correspondió al 'nivel de desarrollo de las habilidades de comprensión lectora', es decir, el grado de logro alcanzado por los estudiantes objeto de estudio en cada una de las pruebas de comprensión lectora de $8^{\circ}$ grado de la batería evaluativa CL-PT; finalmente, la variable independiente se conceptualizó como 'programa de desarrollo de las habilidades de pensamiento crítico', entendida como el conjunto secuenciado de actividades, acotadas en el tiempo, que permite a los estudiantes cuestionar socráticamente un texto con el propósito de analizar y evaluar, en función de estándares y/o criterios, el razonamiento del autor y el razonamiento que se genera en el lector producto de sus intentos de comprensión textual. Se formularon dos hipótesis conceptuales. En la primera de ellas se proyectó una mejora significativa del nivel global de desempeño en comprensión lectora de los estudiantes de octavo grado que participaron en el programa de desarrollo de habilidades de pensamiento crítico; en la segunda, una mejora significativa en el nivel de desarrollo de cada una de las habilidades de comprensión lectora de esos mismos estudiantes. Cada una de estas hipótesis se operacionalizó en dos hipótesis estadísticas (cuatro en total con sus correspondientes hipótesis nulas) con el propósito de contrastar los resultados del grupo experimental con los resultados del grupo control, para el cual no se proyectaron mejoras significativas en sus niveles de comprensión lectora. Los puntajes obtenidos por los sujetos del grupo control y experimental en la prueba CL-PT, fueron procesados por el sistema SPSS para estimar, en cada ítem y dimensión, la media y la desviación estándar de los puntajes bruto y de los porcentajes de logro. Para determinar el nivel de significancia de los resultados obtenidos por los sujetos del grupo experimental después de la intervención y comparar esos resultados con los alcanzados por el grupo control se utilizó la prueba inferencial no paramétrica de Wilcoxon, método estadístico que permite comparar dos grupos relacionados (HernándezSampieri y Mendoza 372).

\subsection{Programa de pensamiento crítico}

El programa de intervención fue diseñado por un lingüista y una profesora de educación diferencial con dilatada trayectoria en desarrollo cognitivo en contextos escolares. Fue precisamente esta última profesional quien aplicó el programa en las clases de la 
Artículo. Ivette Doll, Claudio Parra. "Impacto del desarrollo de habilidades de pensamiento crítico en la comprensión lectora de estudiantes de Enseñanza Básica"

asignatura de Lenguaje y Comunicación en el trascurso procedimientos de un año educativo lectivo entre el mes de marzo y noviembre. Esto implicó que los objetivos de las sesiones debieron ajustarse a los objetivos prescritos en el de estudio de la asignatura, especialmente en términos de conocimientos conceptuales programa y procedimentales.

La metodología general del programa se basó en algunos procedimientos derivados del Metacognitive Awareness Inventory (MAI) de Schraw y Dennison (472-475) y del método de la 'lectura detenida' (Santori y Belfatti 1-8; Boelé 1-4 y Brown y Kappes 3). Ello supuso seguir, entre otros, los siguientes pasos: selección de textos de complejidad creciente, identificación de las estrategias por aplicar (de cuestionamiento socrático y trascendencia textual), aprendizaje de los procedimientos, planificación de los propósitos de la lectura, lectura individual, lectura grupal, aplicación de las estrategias, debate y discusión del producto de la aplicación de las estrategias, monitoreo del proceso de comprensión para detectar aciertos y errores, reparación de los errores y evaluación del logro de objetivos.

El programa se estructuró en función de 3 unidades, 26 sesiones (la primera sesión sirvió como introducción) y 39 horas cronológicas de aplicación. Cada sesión tuvo una duración de 90 minutos. La primera unidad tuvo como objetivo general comprender críticamente un texto a través del uso de preguntas abiertas. La segunda unidad se planificó para que los estudiantes comprendieran críticamente un texto a través de una estrategia de análisis textual, basada en la identificación de conceptos relevantes, en la inferencia de la tesis central y en la formulación de preguntas críticas tipificadas; finalmente, la última unidad consideró la comprensión crítica de textos por medio de la aplicación de la estrategia analítica de los universales del pensamiento de Paul y Elder (Critical Thinking. Tools 57-65 y The Thinker's 14-21).

Para ser coherentes con la concepción multimodal de los textos se usaron distintos formatos y tipos de textos: escritos, estáticos (imágenes, iconografías), dinámicos (cortometrajes), proposicionales (tesis, sintagmas, adagios), narrativos (microcuentos, parábolas o alegorías), textos informativos y textos argumentativos. La progresión de estos recursos se planificó en función de la complejidad del contenido (cantidad de información en términos de líneas y párrafos) y de su abstracción (nivel de posibilidades interpretativas). 
En las sesiones 2 y 3, los estudiantes aprendieron a usar dos reglas estratégicas que les permitieron valorar las preguntas de multisistema y socráticas como deseables para realizar un cuestionamiento socrático (Paul y Elder, The Critical Thinking. Tools 123-141). Estas preguntas tienen como propósito elevar el nivel de comprensión textual, ya que permiten a los estudiantes superar la comprensión superficial para aproximarse a una comprensión más profunda y crítica (Sánchez et al. 63) o pasar de un texto base a un modelo situacional del texto que articula la información textual con el conocimiento previo del lector (Kintsch 809834). En las sesiones 5 a 7 se usaron las mismas reglas para cuestionar microcuentos, pero a nivel de reconstrucción de elementos narrativos que no se hallan en la superficie textual. Este ejercicio apunta al desarrollo de inferencias, habilidad clave en la representación del significado textual, el cual está determinado por los objetivos de lectura. Las sesiones 8 y 9 fueron diseñadas para que los estudiantes elaboraran preguntas que les permitieran discriminar los niveles de comprensión explícito, inferencial-interpretativo y crítico. En las sesiones 10 a 22 se exigía elaborar preguntas en función de una estrategia de análisis textual, diseñada por el equipo del programa, que demandó a los estudiantes: focalizar la atención en información clave de la superficie textual, identificar conceptos relevantes, identificar el propósito del autor, identificar la tesis o idea central del texto y formular preguntas de sistema en conflicto, es decir, abiertas. En la sesión 12 se introdujo una taxonomía interrogativa simple como estrategia para potenciar la elaboración de preguntas críticas que apuntó a interrogar causas, efectos, fines, conceptos y medios. Las sesiones 14 y 15 se transformaron en la llave para acceder a un debate crítico, animado desde la metodología de la discusión filosófica (Lipman, Pensamiento 191-226). En las sesiones 23 a 26 los estudiantes debieron usar la estrategia analítica de los elementos universales del razonamiento propuesta por Paul y Elder (Concepts 3-6).

\section{Resultados y discusión}

La Tabla 1 y la Figura 1 sintetizan los resultados globales de la aplicación de la prueba CL-PT en modalidad de pretest y postest. Conforme a los puntajes obtenidos en el pretest, ambos grupos se sitúan en un nivel de desempeño tipificado en el CL-PT como 'en desarrollo' (41-60\%); por lo tanto, el punto de partida de los dos grupos es similar. La 
Artículo. Ivette Doll, Claudio Parra. "Impacto del desarrollo de habilidades de pensamiento crítico en la comprensión lectora de estudiantes de Enseñanza Básica"

categoría 'en desarrollo' indica que, al inicio del estudio, las necesidades de mejora de la comprensión lectora evidenciadas por los estudiantes del grupo control y experimental son semejantes a las fortalezas que estos experimentan en el área. Después de la intervención, es posible apreciar que el grupo control mantiene su nivel de desempeño; en cambio, el grupo experimental transita hacia un nivel superior tipificado como ‘satisfactorio’ ((61-80\%). Este nivel indica un predominio de las fortalezas por sobre las necesidades de mejora experimentadas por los sujetos del grupo intervenido.

Tabla 1

Comparación de los promedios globales de los puntajes bruto (PB) y de los porcentajes de logro (PL) obtenidos por el grupo control y experimental en el pretest y postest

\begin{tabular}{lcccccc}
\hline & \multicolumn{2}{c}{ Grupo control } & \multicolumn{3}{c}{ Grupo experimental } \\
\hline & PB & PL & N & PB & PL & N \\
\hline Pretest & 30,82 & 55,04 & 53 & 28,14 & 50,26 & 57 \\
\hline Postest & 31,82 & 56,83 & 53 & 36,07 & 64,41 & 57 \\
\hline
\end{tabular}

\section{Figura 1}

Comparación de los niveles globales de desempeño obtenidos por el grupo control y experimental en el pretest y postest

\begin{tabular}{lcccc} 
& \multicolumn{2}{c}{ GRUPO CONTROL } & \multicolumn{2}{c}{ GRUPO EXPERIMENTAL } \\
\cline { 2 - 4 } & \multicolumn{2}{c}{ PRETEST } & POSTEST & \multicolumn{2}{c}{ PRETEST } & \multicolumn{1}{c}{ POSTEST } \\
\hline $\begin{array}{l}\text { Nivel } \\
\text { comprensión } \\
\text { Global }\end{array}$ & En desarrollo & En desarrolllo & En desarrollo & $\begin{array}{l}\text { Desarrollo } \\
\text { satisfactorio }\end{array}$ \\
\hline
\end{tabular}

La Tabla 2 presenta los resultados de la aplicación de la prueba de los rangos con signo de Wilcoxon para el puntaje bruto y el porcentaje de logro del grupo control y experimental. Estos resultados indican que el programa de desarrollo de las habilidades de pensamiento crítico mejora significativamente el nivel global de desempeño de comprensión lectora de los sujetos de estudio. Ello se evidencia en la confirmación de las hipótesis 1 y 2 del estudio y en el rechazo de sus homólogas nulas 01 y 02 . En efecto, el alfa de significancia del puntaje bruto y del porcentaje de logro (0,353 para el primero y 0,351 para el segundo) obtenidos por el grupo control al comparar los resultados del pretest y postest a través de la prueba de Wilcoxon, se traduce en una diferencia que no es estadísticamente significativa; 
por el contrario, el grupo intervenido obtiene un alfa de significancia de 0,000, tanto para el puntaje bruto como para el porcentaje de logro, lo cual se expresa en diferencias estadísticamente significativas.

Tabla 2

Resultados de la aplicación de la prueba de Wilcoxon para puntajes brutos y porcentajes de logro asociados al nivel promedio de comprensión global del grupo control y experimental

\begin{tabular}{lllll}
\cline { 2 - 5 } & \multicolumn{2}{c}{ Puntaje bruto } & Porcentaje de logro \\
\cline { 2 - 5 } & -2 & $\begin{array}{l}\text { Significación } \\
\text { asintótica } \\
\text { bilateral }\end{array}$ & $Z$ & $\begin{array}{l}\text { Significación } \\
\text { asintótica } \\
\text { bilateral }\end{array}$ \\
\hline Grupo control &,$- 929^{\mathrm{b}}$ & 0,353 &,$- 934^{\mathrm{b}}$ & 0,351 \\
\hline $\begin{array}{l}\text { Grupo } \\
\text { experimental }\end{array}$ & $-6,023^{\mathrm{b}}$ & 0,00 & $-6,023^{\mathrm{b}}$ & 0,000 \\
\hline
\end{tabular}

Los resultados coinciden, en cierta medida, con los hallazgos de Esmaili y Motaghizadeh (101-127); Rouijel y Bouziane (114-126); Mohseni et al. (1-22) y Gutiérrez (303-320). Estos investigadores también incorporan experiencias programáticas de estimulación de habilidades de pensamiento crítico que mejoran significativamente la comprensión lectora de los sujetos intervenidos.

En la Tabla 3 se advierten las diferencias positivas y negativas que experimentan los sujetos de ambos grupos en cada una de las dimensiones de la batería CL-PT, una vez rendidos el pretest y postest. La Tabla 4 presenta los resultados de la aplicación de la prueba de Wilcoxon.

Tabla 3

Comparación de los puntajes brutos promedio (PB) y porcentajes de logro promedio (PL) obtenidos por el grupo control y grupo experimental en cada una de las dimensiones de comprensión lectora evaluadas en el pretest $y$ postest

\begin{tabular}{|c|c|c|c|c|c|c|c|c|}
\hline & \multicolumn{4}{|c|}{ Grupo control } & \multicolumn{4}{|c|}{ Grupo experimental } \\
\hline & \multicolumn{2}{|c|}{ Pretest } & \multicolumn{2}{|c|}{ Postest } & \multicolumn{2}{|c|}{ Pretest } & \multicolumn{2}{|c|}{ Postest } \\
\hline & PB & PL & PB & PL & PB & PL & PB & PL \\
\hline C.literal & 2,45 & 81,66 & 2,32 & 77,33 & 2,1 & 70 & 2,49 & 83 \\
\hline C.inferencial & 12,02 & 66,77 & 13,03 & 72,28 & 12,32 & 68,44 & 14,58 & 81 \\
\hline C.critica & 4,58 & 57,25 & 4,35 & 54,37 & 3,46 & 43,25 & 4,61 & 57,62 \\
\hline C.metacognitiva & 3,32 & 49 & 3,92 & 49 & 3,44 & 43 & 5,54 & 69,25 \\
\hline Id estruc. y fun. & 3,01 & 50,16 & 3,46 & 57,66 & 2,75 & 45,83 & 3,87 & 64,5 \\
\hline Reorg.informac. & 2,79 & 46,5 & 2,59 & 43,16 & 2,28 & 38 & 2,73 & 45,5 \\
\hline Manejo código & 2,05 & 29,28 & 2,15 & 30,71 & 1,81 & 25,85 & 2,38 & 34 \\
\hline
\end{tabular}


Artículo. Ivette Doll, Claudio Parra. "Impacto del desarrollo de habilidades de pensamiento crítico en la comprensión lectora de estudiantes de Enseñanza Básica"

Tabla 4

Comparación de los resultados obtenidos por el grupo control y experimental en cada una de las dimensiones de comprensión lectora a partir de la aplicación de la prueba no paramétrica de Wilcoxon.

\begin{tabular}{lcc}
\hline \multicolumn{1}{c}{ Dimensión } & $\begin{array}{c}\text { Significación Asintótica } \\
\text { Bilateral Grupo Control }\end{array}$ & $\begin{array}{c}\text { Significación Asintótica } \\
\text { Bilateral Grupo Experimental }\end{array}$ \\
\hline Comprensión Literal (Ítem 8) & 0,506 & 0,037 \\
\hline $\begin{array}{l}\text { Comprensión Inferencial } \\
\text { (Items 1,2,3,4,5) }\end{array}$ & 0,033 & 0,000 \\
\hline $\begin{array}{l}\text { Comprensión Crítica (Ítems 5 y } \\
11 \text { ) }\end{array}$ & 0,506 & 0,004 \\
\hline $\begin{array}{l}\text { Comprensión Metacognitiva } \\
\text { (Items 7 y 12) }\end{array}$ & 0,990 & 0,000 \\
\hline $\begin{array}{l}\text { Reconocimiento de Estructuras } \\
\text { y Funciones (Ítem 14) }\end{array}$ & 0,034 & 0,000 \\
\hline $\begin{array}{l}\text { Reorganización de la } \\
\text { Información (Ítem 9) }\end{array}$ & 0,477 & 0,076 \\
\hline $\begin{array}{l}\text { Manejo del Código (Ítems 6 y } \\
\text { 13) }\end{array}$ & 0,461 & 0,002 \\
\hline
\end{tabular}

Los resultados permiten confirmar las hipótesis 3 y 4 y rechazar sus correlatos nulos 03 y 04 en el área de 'comprensión literal', es decir, el grupo control presenta diferencias que no son estadísticamente significativas $(0,506)$; en cambio, el experimental obtiene un alfa de significancia de 0,037. De ello se colige que, para la muestra intervenida, el programa de desarrollo de habilidades de pensamiento crítico tiene un efecto positivo en la recuperación de información explícita del texto o en la construcción de un texto base (Kintsch 811) que deriva en niveles de comprensión superficial de los lectores (Sánchez et al. 63). Los resultados a favor del grupo experimental también se ratifican en los tipos de niveles de desempeño que se explicitan en la batería evaluativa CL-PT, conforme a los porcentajes de logro obtenidos en el área de comprensión literal. En efecto, el grupo control experimenta una regresión en sus niveles de desempeño, ya que transita desde un nivel 'muy desarrollado', en el pretest, a un nivel de desarrollo 'satisfactorio', en el postest. El retroceso implica una disminución de las fortalezas evidenciadas en el área de estudio; en cambio, el grupo experimental exhibe un cambio significativo al pasar de un nivel de desarrollo 'satisfactorio' a un nivel 'muy desarrollado'. Este cambio implica que, después de la intervención, en el grupo experimental predominan ampliamente las fortalezas por sobre las necesidades de aprendizaje. Se estima que estos resultados pueden tener su origen en el uso de las estrategias de análisis textual empleadas en la investigación. Al respecto, cabe señalar que la estrategia 
usada en las sesiones 10 a 22 demandó la focalización de los lectores en la información relevante de la superficie textual, con el propósito de anclar la elaboración de las inferencias. Por su parte, la estrategia de análisis de las sesiones 23 a 26 contempló la búsqueda de información relevante y explícita, relacionada fundamentalmente con los conceptos clave del texto y el uso de información declarativa o factual que apoya las ideas o tesis del autor.

En el área de la comprensión inferencial, los resultados del estudio se traducen en el rechazo de la hipótesis de trabajo 3 y en la confirmación de la hipótesis nula 03, ya que el grupo control sí logra una diferencia estadísticamente significativa $(0,033)$ al comparar los resultados del pretest y del postest. Pese a ello, tanto en el pretest como en el postest, mantiene un nivel de desempeño tipificado como desarrollo 'satisfactorio'. Por su parte, los resultados alcanzados por el grupo experimental permiten confirmar la hipótesis 4 de la investigación y rechazar la hipótesis nula 04, es decir, los puntajes logrados por el grupo intervenido en el postest son estadísticamente mejores $(0,000)$ que aquellos alcanzados en el pretest. Esta positiva y significativa diferencia se traduce en el avance de un tramo en el continuo de niveles de desempeños definidos en el manual del CL-PT, en otras palabras, se pasa de un nivel de desarrollo 'satisfactorio' a un nivel 'muy desarrollado', lo que implica un amplio predominio de las fortalezas por sobre las necesidades de aprendizaje. La ganancia que experimenta el grupo intervenido cuando se le compara con el control, permite concluir que los primeros tienen más opciones de mejora de la comprensión inferencial por el hecho de participar en un programa que estimula sus habilidades de pensamiento crítico. Esto implica un mejor desempeño al momento de integrar la información del texto con los conocimientos previos del lector, actividad fundamental para la elaboración de la macroestructura textual (Kintsch 811). Los positivos y significativos resultados relacionados con el efecto del programa en la comprensión inferencial tienen cierta coincidencia con los hallazgos de Din (6-10) y Cojocariu y Butnaru (22-28). El primero de ellos concluye una positiva y significativa correlación entre los resultados obtenidos por estudiantes pakistaníes de bachillerato en una prueba de lectura crítica y los resultados del subtest de inferencias del Whatson-Glaser Critical Thinking Appraisal (Watson y Glaser 2); las otras investigadoras determinan que un grupo de estudiantes rumanos de cuarto grado mejoran, entre otras habilidades, su capacidad inferencial cuando son sometidos a un programa que estimula el pensamiento crítico. En el marco teórico del presente estudio se argumenta a favor de las 
Artículo. Ivette Doll, Claudio Parra. "Impacto del desarrollo de habilidades de pensamiento crítico en la comprensión lectora de estudiantes de Enseñanza Básica"

relaciones de reciprocidad entre el pensamiento crítico y la comprensión lectora, toda vez que ambas variables necesitan de las inferencias para operar sobre los objetos o sujetos que son analizados o evaluados. Para Paul y Elder (The Thinker's 11) la inferencia es una microhabilidad del pensamiento crítico; para Parodi (La Teoría 151), la misma habilidad se constituye en el centro del proceso lector. Por ello, el programa de desarrollo de habilidades de pensamiento crítico del presente estudio centraliza el uso de inferencias en cada una de las estrategias que lo estudiantes aplican en los procesos lectores, especialmente cuando estos se enfrentan a la comprensión de textos alegóricos o deben realizar una lectura multisemiótica de textos dinámicos (cortometrajes, por ejemplo) o estáticos (fotografías o imágenes).

Para la dimensión de la comprensión crítica, los resultados indican que las hipótesis de trabajo 3 y 4 deben ser confirmadas y las nulas 03 y 04 rechazadas, es decir, solo el grupo experimental presenta diferencias estadísticamente significativas entre los puntajes obtenidos en el pretest y aquellos que se alcanzan en el postest $(0,004)$. El alfa de significancia del grupo control $(0,506)$ equivale a una regresión en los puntajes $(-2,88 \%)$. Los logros que el grupo experimental obtiene como producto de la intervención, se traducen, según los fundamentos teóricos del CL-PT, en una leve mejora de la construcción de interpretaciones personales, juicios y análisis de las intenciones del autor, entre otras habilidades. Esta mejora no es suficiente para cambiar el nivel de desempeño definido en el CL-PT, ya que, después de la intervención, el grupo se mantiene en un nivel tipificado como 'en desarrollo', lo que significa que las fortalezas evidenciadas en el área son semejantes a las necesidades de aprendizaje. Parece contradictorio en el estudio que, aun existiendo diferencias estadísticamente significativas entre el pretest y el postest del grupo experimental en la dimensión comprensión crítica, estos resultados no se expresen en un cambio positivo en los niveles de desempeño ('en desarrollo'). Tal vez, esto se deba a la cantidad y al tipo de reactivo que deben enfrentar los estudiantes en el CL-PT, y al aspecto del pensamiento crítico que se evalúa. En efecto, la dimensión solo es evaluada a través de dos preguntas: el ítem 5 es de tipo estructurado y objetivo (selección múltiple), procedimiento que no facilita la expresión o formulación libre de juicios y de preguntas socráticas, y el ítem 11 es semiestructurado (cuadro sinóptico para diferenciar hechos de opiniones), modalidad evaluativa que se centra en un aspecto muy limitado del pensamiento crítico. Parece pertinente indicar que autores 
como Din (6-10) hallan correlaciones positivas entre el pensamiento crítico y la lectura crítica. Pese a ello, la mejora documentada en el presente estudio no es suficiente para apoyar la idea de que la correlación sea alta, ya que tanto los estudiantes del grupo experimental como los del grupo control no mutaron en el nivel de desempeño, manteniéndose ambos grupos en un nivel 'en desarrollo'.

El análisis comparativo de los resultados obtenidos por el grupo experimental y control en la dimensión de comprensión metacognitiva confirma las hipótesis de trabajo 3 y 4 y el rechazo las hipótesis nulas 03 y 04, en otras palabras, solo el grupo intervenido presenta diferencias estadísticamente significativas entre los puntajes del pretest y postest $(0,00)$. El grupo control no evidencia cambios en sus puntajes (ni negativos, ni positivos), lo cual deriva en un alfa de significancia de 0,990. Esta es el área en la cual el grupo experimental logra sus mejores resultados $(26,25 \%$ de ganancia), pasando de un nivel de desempeño 'en desarrollo' a un nivel de 'desarrollo satisfactorio', lo que implica un mejor manejo en la toma de consciencia y regulación de los propios procesos de comprensión. Los cambios evidenciados por el grupo experimental en el área de la comprensión metacognitiva confirman la positiva correlación que, según algunos investigadores, existe entre el pensamiento crítico y la metacognición. Así queda demostrado en los estudios correlacionales efectuados por Arslan (3-6), Diella y Adriansyah (136-139), Cakici (19-124) y Magno (145-152). La correlación entre ambas variables justifica, por lo tanto, la inclusión de la autorregulación, de la autocorrección de los juicios y del monitoreo como conceptos clave en las definiciones que se han elaborado para determinar qué es el pensamiento crítico. Los resultados también confirman la relevancia que le otorgan a la metacognición o a la metacomprensión autores como Peronard et al. (151), Parodi (La Teoría 151) y Zayas (16).

Las diferencias estadísticamente significativas logradas por el grupo control y experimental en el área de la identificación de estructuras y funciones (0,034 para el primero y 0,00 para el segundo) derivan en el rechazo de la hipótesis de trabajo 3 y de la nula 04 y en la confirmación de la hipótesis de trabajo 4 y de la nula 03. Sin perjuicio de lo anterior, se advierte que el grupo intervenido obtiene mejores resultados como consecuencia de su participación en el programa de desarrollo de habilidades de pensamiento crítico, lo que le permite pasar de un nivel de desempeño ‘en desarrollo' a un nivel de 'desarrollo satisfactorio' (predominio de las fortalezas por sobre las necesidades de aprendizaje). Es probable que la 
Artículo. Ivette Doll, Claudio Parra. "Impacto del desarrollo de habilidades de pensamiento crítico en la comprensión lectora de estudiantes de Enseñanza Básica"

diferencia a favor del grupo experimental se deba al uso de distintos tipos de textos en las tareas de comprensión lectora que los sujetos debieron enfrentar y al reiterado emplazamiento para que el lector infiriera el propósito del autor, tarea que se facilita cuando se identifica el tipo de estructura a partir de la cual se organiza el contenido del texto. Al respecto, parece conveniente recordar que el proceso inferencial está determinado, entre otros factores, por la estructura de un texto (Parodi, La Comprensión 58).

A partir de las diferencias entre los puntajes de pretest y postest, logrados por ambos grupos en la dimensión de reorganización de la información se concluye que tanto la hipótesis 3 como la 4 deben ser rechazadas; por el contrario, se confirman las hipótesis nulas 03 y 04. A pesar de que ambos grupos obtienen diferencias que no son estadísticamente significativas $(0,477$ el control y 0,076 el experimental) se consigna que solo el grupo experimental cambia su desempeño al pasar de un nivel 'emergente' a un nivel 'en desarrollo'. Ello implica que experimenta una leve mejoría en su capacidad de síntesis como consecuencia de su participación en el programa de desarrollo de las habilidades de pensamiento crítico.

Los resultados alcanzados por ambos grupos en la dimensión manejo del código permiten confirmar las hipótesis de trabajo 3 y 4 y rechazar las nulas 03 y 04, en otras palabras, solo el grupo experimental logra diferencias estadísticamente significativas al comparar los puntajes del pretest y del postest. Pese a ello, este grupo se mantiene en un nivel de desempeño 'emergente', lo que implica un predominio de las necesidades de aprendizaje por sobre las fortalezas. Es importante destacar que los ejercicios del programa de intervención se centraron solo en el fortalecimiento de uno de los tantos aspectos lingüísticos contemplados en el CL-PT, esto es, el reconocimiento y manejo de palabras.

\section{Conclusiones}

Los hallazgos permiten establecer que el desarrollo programático de las habilidades de pensamiento crítico, a través del uso de estrategias que centralizan la interrogación textual de naturaleza socrática, produce una mejora significativa en el nivel global de desempeño de comprensión lectora de los estudiantes objeto de estudio, así como también en las habilidades fundamentales que enmarcan el procesamiento textual. 
Para transferir este tipo de prácticas a nivel de aula, es necesario superar las concepciones teóricas que reducen los niveles críticos de comprensión solo a la reflexión acerca del contenido o la forma textual o a la evaluación de la calidad y validez de la información, tal como lo propone el sistema de evaluación PISA. La comprensión textual es mucho más que aquello. Por un lado, es un macroproceso de pensamiento regulado por el pensamiento crítico en tanto pensamiento de orden superior que posibilita analizar y evaluar el razonamiento de quien produce los significados textuales (el autor o la autora) y el propio razonamiento de quien los interpreta (el lector o la lectora) para efectos de crear sus propios significados; por el otro, es preciso asumir que todo proceso lector de orden superior debe tener como finalidad pensar críticamente. Estas simples afirmaciones tienen significativas implicancias desde la perspectiva de la didáctica de la comprensión textual, ya que las intervenciones del proceso lector debiesen encaminarse a estimular la creación de nuevos significados y, de manera recursiva, al mejoramiento del razonamiento. Por ello, profesoras y profesoras deben perfeccionar sus talentos didácticos para enseñar a pensar por medio de la lectura comprensiva y viceversa. Junto con esto, deben desprenderse de aquellas concepciones metodológicas que fragmentan artificialmente la enseñanza de la comprensión lectora.

\section{REFERENCIAS}

Ardhian, Trio, Inayatul Ummah, Siti Anafiah y Reza Rachmadtulla. "Reading and Critical Thinking Techniques on Understanding Reading Skills for Early Grade Students in Elementary School”. International Journal of Instruction, vol. 13, núm. 3, 2020, pp. 107-118.

Arslan, Serhat. "Investigating Predictive role of Critical Thinking on Metacognition with Structural Equation Modeling". The Malaysian Online Journal of Educational Science, vol. 3, núm. 2, 2015, pp. 1-10.

Boelé. Amy. "Text-Dependent Questions. Reflecting and Trascending the Text". Reading Teacher, vol. 70, núm. 2, 2016, pp. 207-220.

Brown, Sheila y Lee Kappes. Implementing the Common Core State Standards: A Primer on Close Reading of Text. The Aspen Institute, 2012.

Cairney, Trevor. Enseñanza de la comprensión lectora, 6 ${ }^{\mathrm{a}}$ ed. Morata, 2018.

Çakıci, Dilek. "Metacognitive Awareness and Critical Thinking Abilities of Pre-service EFL Teachers". Journal of Education and Learning, vol. 7, núm. 5, 2018, p. 116-129.

Cohen, Louis, Lawrence Manion y Keith Morrison. Research methods in education, $6^{\mathrm{a}}$. ed. Routledge, 2007. 
Artículo. Ivette Doll, Claudio Parra. "Impacto del desarrollo de habilidades de pensamiento crítico en la comprensión lectora de estudiantes de Enseñanza Básica"

Cojocariu, Venera y Carmen Butnaru. "Asking questions-critical thinking tools". ProcediaSocial and Behavioral Sciences, núm. 128, 2014, pp. 22-28.

Din, Muhammad. "Evaluating university students' critical thinking ability as reflected in their critical reading skill: A study at bachelor level". Thinking Skills and Creativity, núm.35, 100627, 2020, pp. 1-11.

Diella, Dea y Ryan Adriansyah. "The Correlation of Metacognition with Critical Thinking Skills of Grade XI Students on Human Excretion System Concept". Journal Penelitian dan Pembelajaran IPA, vol. 3, núm. 2, 2017, pp. 134-142.

Esmaili, Sajjad e Isa Motaghizadeh. "An investigation the effect of critical thinking skills on reading comprehension of literary Arabic texts among Iranian Language and Literature Arabic students". Language Related Research, vol.8, núm. 2, 2017, pp. 101-127.

Facione, Peter. Critical Thinking: A Statement of Expert Consensus for Purposes of Educational Assessment and Instruction. Academic Press, 1998.

Fernández, Nelly, Carlos Mayora y Rubena St. Louis. "Pensamiento crítico y comprensión de la lectura en un curso de inglés como lengua extranjera". Íkala, Revista de Lenguaje y Cultura, vol. 21, núm. 1, 2016, pp.15-29.

Flavell, John. "Metacognitive aspects of problem solving". The nature of intelligence, edición de Lauren Resnick. Lawrence Erlbaum, 1976, pp. 231-235.

Freire, Paulo y Antonio Faúndez, Antonio. Por una pedagogía de la pregunta. Crítica a una educación basada en respuestas a preguntas inexistentes, $5^{\mathrm{a}}$ ed. Siglo veintiuno, 2013.

Fundación Arauco, Alejandra Medina y Ana María Gajardo. Pruebas de Comprensión Lectora y Producción de Textos (CL-PT). Ediciones UC, 2013.

Gutiérrez, Raúl. "Efectos de la lectura dialógica en la mejora de la comprensión lectora de estudiantes de Educación Primaria”. Revista de Psicodidáctica, vol.21, núm. 2, 2016, pp.303-320.

Hernández Sampieri, Roberto y Christian Mendoza. Metodología de la investigación: las rutas cuantitativa, cualitativa y mixta. MacGraw Hill, 2018.

Kintsh, Walter. "Revisiting the Construction-Integration Model of Text Comprehension and Its Implications for Instruction". Theorical Models and Processes of Reading, edición de Dona Alvermann, Norman Unrau y Robert Rudell. International Reading Association, 2013, pp.807-839.

Lipman, Mattew, Anne Sharp y Frecerick Oscaya. La filosofía en el aula, $3^{\mathrm{a}}$ ed. De la Torre, 2002.

Lipman, Matthew. Pensamiento complejo y educación, $1^{\mathrm{a}}$ ed. EPUB. De la Torre, 2014.

Magno, Carlos. "The role of metacognitive skills in developing critical thinking". Metacognition Learning, vol.5, núm. 2, 2010, pp. 137-156.

Mohseni, Fotemeh, Zohereh Seifoori y Saeideh Ahangari. "The impact of metacognitive strategy training and critical thinking awareness-raising on reading comprehension". Cogent Education, núm. 7, 2020, pp.1-22.

Núñez, María Pilar. "La comprensión lectora: aspectos teóricos y didácticos". Didáctica de la lengua y la literatura, coordinación de Juan Mata, María Pilar Núñez y José Rienda. Pirámide, 2015, pp. 114-152. 
Organización para la Cooperación y el Desarrollo Económico. 21st-Century Readers. Developing Literacy Skills in a Digital Words. OECD, 2016.

Paul, Richard y Elder, Linda. The Thinker's Guide to Analytic Thinking, 2a ed. Foundation for Critical Thinking, 2017.

---. Critical Thinking. Concepts and Tools, $7^{\mathrm{a}}$. ed. Foundation for Critical Thinking, 2016.

---. Critical Thinking. Tools for Taking Charge of your Learning and your Life, $3^{\mathrm{a}}$. ed. Pearson, 2015.

---. "Critical Thinking: The Art of Socratic Questioning". Journal of Developmental Education, vol. 31, núm. 1, 2007, pp. 36-37.

Parodi, Giovani. "Multisemiosis y lingüística de corpus: artefactos multisemióticos en los textos de seis disciplinas en el corpus PUCV-2010”. Revista de Lingüística Teórica y Aplicada, vol. 48, núm. 2, 2010, pp.33-70.

---. "La Teoría de la Comunicabilidad: Notas para una concepción integral de la comprensión de textos escritos. Revista Signos, vol. 44, núm. 76, 2011, pp. 146-167.

---. La comprensión de textos escritos. La teoría de la comunicabilidad. Eudeba, 2014.

Parodi, Giovanni, Marianne Peronard y Romualdo Ibéñez.. Saber leer. Aguilar, 2010.

Peronard, Mariane, Nina Crespo e Inés Guerrero. "El conocimiento metacomprensivo en escolares chilenos de Educación Básica”. Revista Signos, vol. 34, núm. 49-50, 2001, pp.149-164.

Rouijel, El Hassan y Abdelmajid Bouziane,. "The effect of explicit instruction in critical thinking on higher-order thinking skills in reading comprehension: an experimental study". European Journal of English Language Teaching, vol. 5, núm. 1, 2019, pp. 114-126.

Sánchez, Emilio, Ricardo García y Javier Rosales. La lectura en el aula. Qué se hace, qué se debe hacer y qué se puede hacer. Graó, 2010.

Santori, Diana y Mónica Belfatti. "Do Text-Dependent Questions Need to Be TeacherDependent? Close Reading From Another Angle”. The Reading Teacher, vol. 70, núm. 6, 2016, pp. 649-665.

Schraw, Gregory y Rayne Dennison. "Assessing Metacognitive Awareness". Contemporary Educational Psychology, vol. 19, núm. 5, 1994, pp. 460-75.

Watson, Goodwin y Edwin Glaser, Edwin. Whatson-Glaser Critical Thinking Appraisal UK Edition, $5^{\text {a }}$. ed. Pearson, 2002.

Zayas, Felipe. 10 ideas clave. La competencia lectora según PISA. Reflexiones y orientaciones didácticas. Graó, 2012 